\title{
Clinical comparative investigation of efficacy and toxicity of cisplatin plus gemcitabine or plus Abraxane as first-line chemotherapy for stage III/IV non-small-cell lung cancer
}

\author{
This article was published in the following Dove Press journal: \\ OncoTargets and Therapy \\ 16 September 2016 \\ Number of times this article has been viewed
}

\author{
Dan $A i^{1,2}$ \\ Yan Guan² \\ Xiu-Ju Liu² \\ Chu-Feng Zhang ${ }^{1,2}$ \\ Peng Wang ${ }^{1,2}$ \\ Hong-Lu Liang ${ }^{1,2}$ \\ Qi-Sen Guo ${ }^{2}$
}

'School of Medicine and Life Sciences, University of Jinan-Shandong Academy of Medical Sciences, China; ${ }^{2}$ Shandong Cancer Hospital affliated to Shandong University, Shandong Academy of Medical Sciences, Jinan, Shandong, China

Correspondence: Qi-Sen Guo Shandong Cancer Hospital affiliated to Shandong University, Shandong Academy of Medical Sciences, 440 Jiyan Road, Jinan 2501 I7, Shandong, China

Tel +86 I5624065976

Email mI5624065976@I63.com
Purpose: The purpose of this study was to observe the clinical efficacy and toxicity of cisplatin in combination with gemcitabine or Abraxane as first-line chemotherapy for stage III/IV nonsmall-cell lung cancer (NSCLC).

Patients and methods: A total of 200 patients with advanced NSCLC, which was confirmed by pathology or cytology, were enrolled into our research by reviewing previous complete and retrievable medical records data of our hospital. A total of 100 patients were treated with gemcitabine $\left(1,000 \mathrm{mg} / \mathrm{m}^{2}\right.$, day 1 and day 8$)$ in combination with cisplatin $\left(75 \mathrm{mg} / \mathrm{m}^{2}\right.$, days $1-3$; GP group) and another 100 patients were treated with Abraxane $\left(260 \mathrm{mg} / \mathrm{m}^{2}\right.$, day 1) in combination with cisplatin ( $75 \mathrm{mg} / \mathrm{m}^{2}$, days $1-3$; TP group). Twenty-one days were required to complete one cycle; at least two cycles were completed by each group.

Results: For the 100 patients in the GP group, the effective response rate (RR) was $27 \%$, the disease control rate (DCR) was $63 \%$, and the median progression-free survival (PFS) time was 8 months. For the 100 patients in the TP group, the RR was $52 \%$, the DCR was $75 \%$, and the median PFS was 20 months. There was significant difference in $\mathrm{RR}(P<0.001)$, but no significant difference in DSR and PFS $(P>0.05)$. Common treatment-related adverse events were hematologic toxicity and gastrointestinal reaction. Hematologic toxicity mainly included decreased white blood cells and platelets. The differences between the two groups were statistically significant $(P<0.05)$. Gastrointestinal reaction mainly included nausea and vomiting. There was no statistical significance between them $(P=0.805)$. For the 85 patients with squamous carcinoma in the TP group, the RR was $60 \%$, the DCR was $78 \%$, and the median PFS was 7.5 months. For the 85 patients with squamous carcinoma in the GP group, the RR was $36 \%$, the DCR was $62 \%$, and the median PFS was 18.5 months. There was significant difference in RR $(P=0.024)$, but no significant difference in DSR and PFS $(P>0.05)$. For the 115 patients with adenocarcinoma in the TP group, the RR was $47 \%$, the DCR was $73 \%$, and the median PFS was 8 months. For the 115 patients with adenocarcinoma in the GP group, the RR was $20 \%$, the DCR was $64 \%$, and the median PFS was 20.5 months. There was significant difference in RR $(P=0.003)$, but no significant difference in DCR and PFS $(P>0.05)$.

Conclusion: The efficacy of cisplatin in combination with Abraxane is better than that with gemcitabine in the treatment of NSCLC, and the treatment has less risk of hematologic toxicity.

Keywords: cisplatin, Abraxane, gemcitabine, advanced non-small-cell lung cancer, chemotherapy

\section{Introduction}

Lung cancer is one of the most common malignant tumors, of which advanced non-small-cell lung cancer (NSCLC) accounts for $\sim 80 \%-85 \%{ }^{1}$ Nearly $75 \%-80 \%$ of 
NSCLC patients are diagnosed at an advanced stage. With poor sensitivity to chemotherapy, the remission rate is only $15 \%-20 \%$ in the $1970 \mathrm{~s}$, and the average 5 -year survival rate for NSCLC patients is only $15 \% .^{2}$ In the recent 10 years, the curative effect of chemotherapy has increased significantly and the remission rate has reached up to $40 \%$ because of the continuous increase in new effective anticancer drugs and new schemes in both quantity and quality. Chemotherapy is one of the most important treatment methods, especially for advanced NSCLC. ${ }^{3}$ We conducted clinical retrospective observational analyses to observe the curative effect and toxicity of cisplatin in combination with gemcitabine or Abraxane as first-line chemotherapy for stage III/IV NSCLC.

\section{Patients and methods}

\section{Clinical data}

We retrospectively analyzed 200 patients with advanced NSCLC who were treated in our hospital from May 2012 to October 2015. Each case abided by the following principles: advanced NSCLC (stage III or IV) was confirmed by pathology or cytology, no second primary tumor or history of other tumors was observed, had previously untreated NSCLC and received at least two cycles of cisplatin plus gemcitabine or cisplatin plus Abraxane, had least one measurable lesion, had no mutations, 20 years $\leq$ age at diagnosis $\leq 75$ years, had Eastern Cooperative Oncology Group (ECOG) score 0-3, and possessed complete clinical data, including sex, age, pathological data, ECOG score, treatment, and follow-up information. There was significant difference in these data $(P>0.05)$. The CONSORT diagram reveals data collection. A total of 455 subjects were screened and 200 were enrolled (Figure 1). Table 1 lists the general characteristics of the 200 cases.

\section{Methods}

\section{GP group}

Gemcitabine was used at a dose of $1,000 \mathrm{mg} / \mathrm{m}^{2}$ on day 1 and day 8 , and cisplatin was administered on days 1-3 of each course at a dose of $75 \mathrm{mg} / \mathrm{m}^{2}$. They were added to $250 \mathrm{~mL}$ saline, respectively, and were used by the method

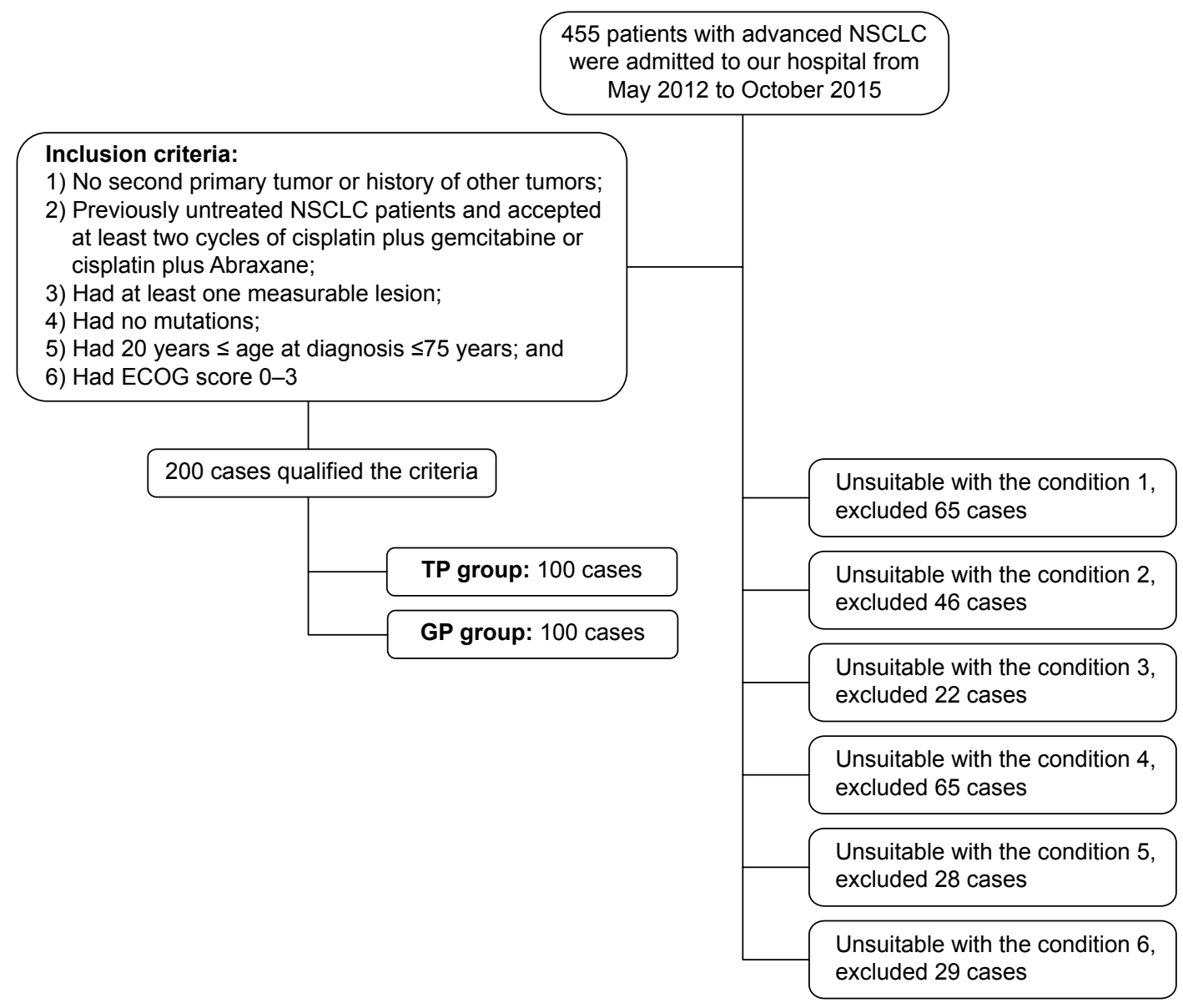

Figure I The CONSORT diagram.

Abbreviations: NSCLC, non-small-cell lung cancer; ECOG, Eastern Cooperative Oncology Group. 
Table I Baseline characteristics of 200 patients

\begin{tabular}{llll}
\hline Characteristic & GP group & TP group & P-value \\
\hline Sex, $\mathrm{n}$ & & & 0.428 \\
$\quad$ Male & 75 & 70 & \\
$\quad$ Female & 25 & 30 & \\
Age, years & & & 0.465 \\
$\quad$ Median & 58.5 & 57.8 & \\
$\quad<65$ & 65 & 60 & \\
$\geq 65$ & 35 & 40 & 0.474 \\
Pathology type, $\mathrm{n}$ & & & \\
$\quad$ Squamous carcinoma & 45 & 40 & \\
$\quad$ Adenocarcinoma & 55 & 60 & \\
ECOG scores, $\mathrm{n}$ & & & \\
0-I & 75 & 80 & \\
2-3 & 25 & 20 & 0.450 \\
Clinical stage, $\mathrm{n}$ & & & \\
III & 65 & 70 & \\
IIIA & 40 & 40 & \\
IIIB & 25 & 30 & \\
IV & 35 & 30 &
\end{tabular}

Notes: GP group refer to the group of patients treated with gemcitabine in combination with cisplatin. TP group refer to the group of patients treated with abraxane in combination with cisplatin.

Abbreviation: ECOG, Eastern Cooperative Oncology Group.

of intravenous (iv) drip. In most instances, cisplatin infusion was preceded by parenteral administration of 5-HT3 receptor antagonists. No prophylactic antiemetics were routinely used on the days of gemcitabine administration.

\section{TP group}

Abraxane was used at a dose of $260 \mathrm{mg} / \mathrm{m}^{2}$ on day 1 ; it was also added to $250 \mathrm{~mL}$ of normal saline and was used by the method of iv drip. Electrocardiographic monitoring was performed during the treatment of patients. In order to prevent allergic reactions, 30 minutes before the therapy, iv dexamethasone $20 \mathrm{mg}$, intramuscular injection Phenergan $50 \mathrm{mg}$, and iv cimetidine $400 \mathrm{mg}$ were used. The usage of cisplatin was as described earlier.

The medicines were used appropriately to stop vomiting, protect liver and stomach, and enhance immunity in the two groups. Routinely, 5-HT receptor antagonist was used during chemotherapy to stop vomiting. According to the result of blood routine examination, the recombinant human granulocyte colony stimulating factor was timely used $(2 \mu \mathrm{g} / \mathrm{kg} / \mathrm{d}$, subcutaneous injection) when the white blood cell count was reduced to $\leq 2.0 \times 10^{9} / \mathrm{L}$.

\section{Ethical approval}

All procedures performed in studies involving human participants were approved by the institutional review board of the Shandong Cancer Hospital and
Institute. Written informed consent was obtained from every patient.

\section{Assessment of efficacy and adverse reactions}

The objective efficacy was evaluated using the Response Evaluation Criteria in Solid Tumors Version 1.1. The observation indicator included complete remission (CR). All visible lesions disappeared completely. The partial response (PR) was the sum of the longest diameter of tumor lesions reduced by $>30 \%$. The stable disease (SD) was between PR and progressive disease (PD). Disease development (PD) was the longest diameter of tumor lesions increased to $20 \%$ or with new lesions. The effective response rate (RR) was $\mathrm{CR}+\mathrm{PR}$. The disease control rate (DCR) was $\mathrm{CR}+\mathrm{PR}+\mathrm{SD}$. The progression-free survival (PFS) was the time from the first medication to the first objective progression of disease. According to World Health Organization's anticancer drug toxicity response evaluation criteria, the adverse reactions were divided into 0-IV degrees.

\section{Follow-up visits}

Telephonic or outpatient follow-up was adopted, and the adverse reactions, condition changes of patients, and time of death were recorded. Lost to follow-up was defined as patients and their families could not be contacted by telephone or outpatient follow-up. The last follow-up time of this study was on March 20, 2016.

\section{Statistics analysis}

Statistical analysis was done by SPSS 17.0 software. Correlation between clinical features and curative effects was valued by using $\chi^{2}$ test. PFS was analyzed using KaplanMeier methods, and the differences between groups were compared by log-rank test. A value of $P<0.05$ was considered to be statistically significant.

\section{Results \\ Short-term efficacy}

A total of 200 patients with advanced NSCLC were enrolled into this study. All could participate in the evaluation of clinical curative effect. Both groups completed the chemotherapy within two to eight cycles. In the TP group, the RR was $52 \%$ (95\% CI: $42 \%-62 \%)$ and DCR was $75 \%$ (95\% CI: 67\%-83\%) and five patients achieved CR, 47 achieved PR, 23 reached a stage of SD, and 25 suffered from PD. In the GP group, the RR was $27 \%$ (95\% CI: $19 \%-35 \%)$ and DCR was $63 \%$ (95\% CI: 54\%-72\%) and two patients achieved CR, 
25 achieved PR, 36 reached a stage of SD, and 37 suffered from PD. There was significant difference in $\mathrm{RR}(P=0.024)$, but no significant difference in DCR $(P>0.05)$.

The RRs of patients with squamous carcinoma in the GP group and TP group were 36\% (95\% CI: $22 \%-50 \%$ ) and $60 \%$ (95\% CI: $45 \%-75 \%$ ), respectively. There was significant difference between them $(P<0.05)$. The DCRs were 62\% (95\% CI: 51\%-73\%) and 78\% (95\% CI: 65\%-91\%), respectively. There was no significant difference $(P>0.05)$. The RRs of patients with adenocarcinoma in the GP group and TP group were $20 \%$ (95\% CI: $9 \%-31 \%$ ) and $47 \%$ (95\% CI: $37 \%-60 \%$ ), respectively. There was significant difference between them $(P<0.05)$. The DCRs were $64 \%(95 \%$ CI: $51 \%-77 \%$ ) and $73 \%$ (95\% CI: 62\%-84\%), respectively. There was no significant difference between them $(P>0.05)$. Tables 2-4 list the results.

\section{Long-term survival analysis}

The median PFS time of all 200 patients in the TP group was 20 months (95\% CI: $12-28$ months), which is superior to that in the GP group with 8 months (95\% CI: 7-9 months). There was no significant differences between the two groups $(P>0.05$; Figure 2$)$.

The median PFS time of patients with squamous carcinoma was 18.5 months (95\% CI: 10-27 months) in the TP group and 7.5 months (95\% CI: 6-8 months) in the GP group. There was no significant differences between the two groups ( $P>0.05$; Figure 3$)$.

The median PFS time of patients with adenocarcinoma was 20.5 months (95\% CI: 13-29 months) in the TP group and 8 months ( $95 \%$ CI: 7-9 months) in the GP group. There was no significant differences between the two groups $(P>0.05$; Figure 4).

\section{Toxic and side effect}

The most common adverse reactions were hematologic toxicity and gastrointestinal reaction. Hematologic toxicity mainly included decreased white blood cells and platelets,

Table 2 Analysis of curative effect in two groups

\begin{tabular}{lllllll}
\hline & CR & PR & SD & PD & RR (\%) & DCR (\%) \\
\hline GP & 2 & 25 & 36 & 37 & 27 & 63 \\
TP & 5 & 47 & 23 & 25 & 52 & 75 \\
$P$-value & & & & & $<0.001$ & 0.067 \\
\hline
\end{tabular}

Notes: GP group refer to the group of patients treated with gemcitabine in combination with cisplatin. TP group refer to the group of patients treated with abraxane in combination with cisplatin.

Abbreviations: $C R$, complete remission; PR, partial response; SD, stable disease; $P D$, progressive disease; $R R$, response rate; $D C R$, disease control rate.
Table 3 Analysis of curative effect in squamous carcinoma

\begin{tabular}{lllllll}
\hline & CR & PR & SD & PD & RR (\%) & DCR (\%) \\
\hline GP & 1 & 15 & 12 & 17 & 36 & 62 \\
TP & 2 & 22 & 7 & 9 & 60 & 78 \\
$P$-value & & & & & 0.024 & 0.127 \\
\hline
\end{tabular}

Notes: GP group refer to the group of patients treated with gemcitabine in combination with cisplatin. TP group refer to the group of patients treated with abraxane in combination with cisplatin.

Abbreviations: $C R$, complete remission; PR, partial response; SD, stable disease; $\mathrm{PD}$, progressive disease; $\mathrm{RR}$, response rate; $\mathrm{DCR}$, disease control rate.

and it was statistically significant between the two groups $(P<0.05)$. Gastrointestinal reaction mainly included nausea and vomiting, and there was no statistical significance between them $(P>0.05)$. Table 5 lists the results.

\section{Discussion}

In recent years, a number of studies have confirmed that chemotherapy can prolong the survival of advanced non-small-cell carcinoma and improve the quality of life compared with the best supportive treatments. ${ }^{4,5,7,11,14,15}$ In order to improve the effectiveness of chemotherapy, the domestic and overseas scholars have made a lot of clinical researches. At present, platinum-based regimen is used as a standard regimen for advanced NSCLC. ${ }^{5}$ Moreover, a large number of reports also confirmed that the doublet is superior to a single agent or triple combination..$^{2,4,515}$ Clinical studies have shown that paclitaxel has been approved for the treatment of advanced NSCLC with a broad spectrum of anticancer activity. ${ }^{6-10}$ Fang et al ${ }^{11}$ conducted a clinical study of Abraxane plus platinum as first-line chemotherapy for stage III/IV squamous NSCLC and verified that paclitaxel can reduce the risk of hypersensitivity reactions and blood toxicity induced by organic solvents when used in combination with albumin.

Abraxane is one of the most effective drugs for advanced non-small cell carcinoma. It is a new kind of albumin solvent nanoparticle paclitaxel. To carry paclitaxel with the help of human serum albumin and activate the caveolin on the cell membrane, paclitaxel is transported into the tumor

Table 4 Analysis of curative effect in adenocarcinoma

\begin{tabular}{lllllll}
\hline & CR & PR & SD & PD & RR (\%) & DCR (\%) \\
\hline GP & 1 & 10 & 24 & 20 & 20 & 64 \\
TP & 3 & 25 & 16 & 16 & 47 & 73 \\
P-value & & & & & 0.003 & 0.263 \\
\hline
\end{tabular}

Notes: GP group refer to the group of patients treated with gemcitabine in combination with cisplatin. TP group refer to the group of patients treated with abraxane in combination with cisplatin.

Abbreviations: $\mathrm{CR}$, complete remission; $\mathrm{PR}$, partial response; $\mathrm{SD}$, stable disease; $\mathrm{PD}$, progressive disease; RR, response rate; DCR, disease control rate. 


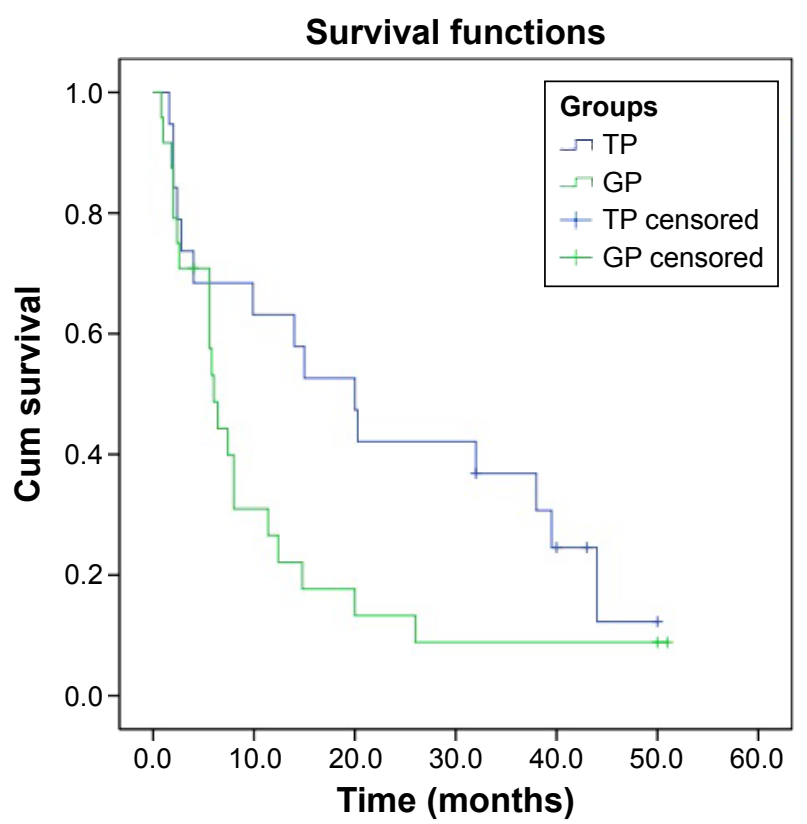

Figure 2 The median PFS time of all 200 patients.

Notes: GP group refer to the group of patients treated with gemcitabine in combination with cisplatin. TP group refer to the group of patients treated with abraxane in combination with cisplatin.

Abbreviations: PFS, progression-free survival; Cum, cumulative.

by vascular endothelial cells. Its mechanism of action is to prevent tumor cell mitosis by interfering with the microtubule system. ${ }^{12}$ Abraxane will have greater antitumor activity with the increase in intratumoral concentrations. ${ }^{13}$ Clinically, it is usually used in combination with platinum. There are a

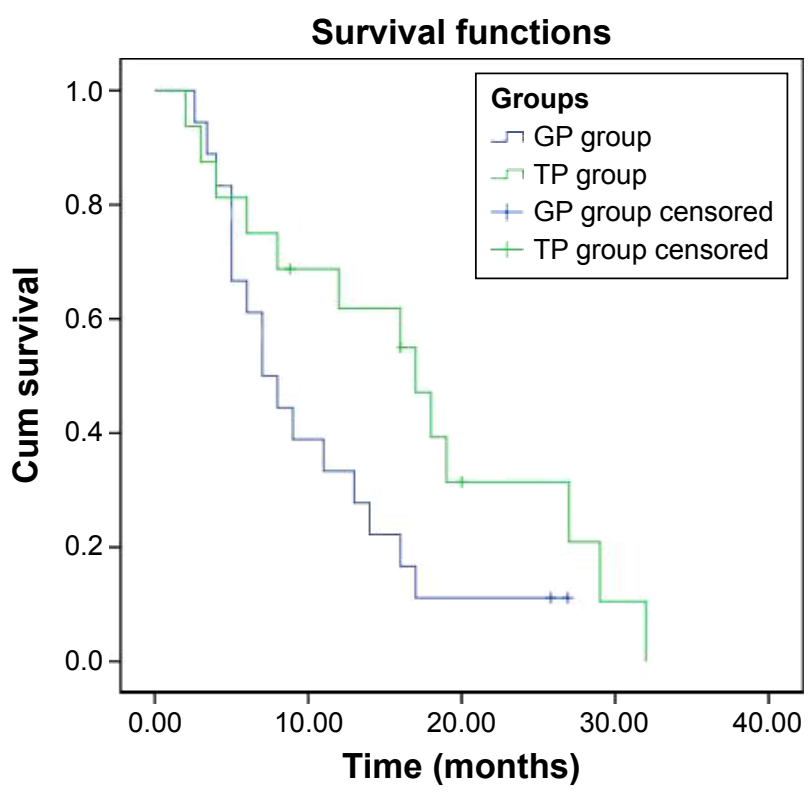

Figure 3 The median PFS time of squamous carcinoma patients.

Notes: GP group refer to the group of patients treated with gemcitabine in combination with cisplatin. TP group refer to the group of patients treated with abraxane in combination with cisplatin.

Abbreviations: PFS, progression-free survival; Cum, cumulative.
Survival functions

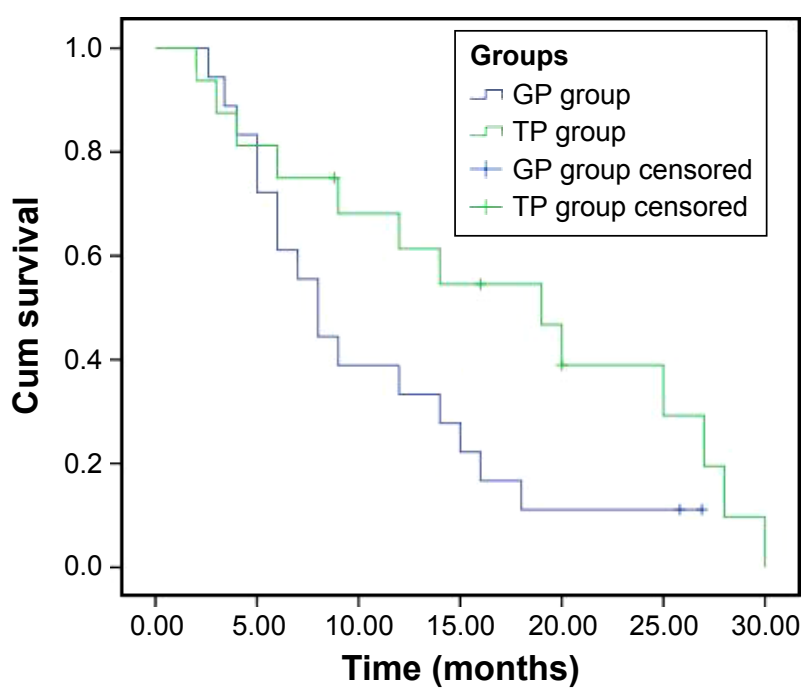

Figure 4 The median PFS time of adenocarcinoma patients.

Notes: GP group refer to the group of patients treated with gemcitabine in combination with cisplatin. TP group refer to the group of patients treated with abraxane in combination with cisplatin.

Abbreviations: PFS, progression-free survival; Cum, cumulative.

lot of reports about the clinical observations of gemcitabine plus cisplatin for treating advanced NSCLC. ${ }^{14}$ However, the comparison of two groups is rare at home and abroad. Schiller et $\mathrm{al}^{15}$ reported about ECOG 1594 clinical trial that made a comparative study about four chemotherapy regimens, including the two groups of cisplatin plus paclitaxel and cisplatin plus gemcitabine. It had no statistically significant differences in curative effects and adverse reactions. The RRs were $22 \%$ and $22 \%$, respectively. One-year survival rates were $31 \%$ and $36 \%$, respectively. What we have researched was Abraxane plus cisplatin and gemcitabine plus cisplatin, and their RRs were higher than ECOG 1594 clinical trials. The RR between the TP group and GP group also had significant difference. The usage of gemcitabine in ECOG 1594 was on the first day, the eighth day, and the 15th day, but the usage in our GP group was only on the first day and the

Table 5 Adverse effects of two groups

\begin{tabular}{lllllll}
\hline Project & \multicolumn{2}{c}{ TP group } & & \multicolumn{2}{c}{ GP group } & P-value \\
\cline { 2 - 3 } & III & IV & & III & IV & \\
\hline Leukopenia, $\mathrm{n}$ & $\mathrm{I} 5$ & 0 & 28 & 5 & 0.003 \\
Thrombocytopenia, $\mathrm{n}$ & $\mathrm{I}$ & 3 & 38 & 7 & $0.00 \mathrm{I}$ \\
Nausea and vomiting, $\mathrm{n}$ & $3 \mathrm{I}$ & 5 & 32 & 2 & 0.767 \\
Liver function damage, $\mathrm{n}$ & $\mathrm{I}$ & 0 & 2 & $\mathrm{I}$ & 0.312 \\
Hair loss, $\mathrm{n}$ & $5 \mathrm{I}$ & 0 & 45 & 0 & 0.396 \\
Muscle and joint pain, $\mathrm{n}$ & 5 & 0 & 3 & 0 & 0.470 \\
\hline
\end{tabular}

Notes: GP group refer to the group of patients treated with gemcitabine in combination with cisplatin. TP group refer to the group of patients treated with abraxane in combination with cisplatin. 
eighth day, which may be the reason for the difference in the curative effect. However, if we increase the dose intensity, the toxicity will increase accordingly. Whether the toxicity and efficacy will change on adding an additional drug on the 15 th day needs to be further observed.

\section{Conclusion}

We recommend cisplatin plus Abraxane as first-line chemotherapy for stage III/IV NSCLC.

\section{Acknowledgment}

This research was supported by Shandong Provincial Natural Science Foundation, People's Republic of China (ZR2015HM044).

\section{Disclosure}

The authors report no conflicts of interest in this work.

\section{References}

1. Barzi A, Pennell NA. Targeting angiogenesis in non-small cell lung cancer: agents in practice and clinical development. Eur J Clin Med Oncol. 2010;2(1):31-42.

2. Molina JR, Yang P, Cassivi SD, Schild SE, Adjei AA. Non-small cell lung cancer epidemiology, risk factors, treatment and survivorship. Mayo Clin Proc. 2008;83(5):584-594.

3. Qiao D, Wang Z, Lu Y, Wen X, Li H, Zhao H. A retrospective study of risk and prognostic factors in relation to lower respiratory tract infection in elderly lung cancer patients. Am J Cancer Res. 2015;5(1): 423-432.

4. Spigel DR, Greco FA. Chemotherapy in metastatic and locally advanced non-small cell lung cancer. Semin Surg Onco. 2003;21(2):98-110.
5. Pujol JL, Quantin X, Choma D, Jacot W, Khial F. Combination chemotherapy without cisplatin in the treatment of advanced non-small cell lung cancer. Lung Cancer. 2003;38(3):S57-S60.

6. Li XD, Han JC, Zhang YJ, et al. Common variations of DNA repair genes are associated with response to platinum-based chemotherapy in NSCLCs. Asian Pac J Cancer Prev. 2013;14(1):145-148.

7. Natukula K, Jamil K, Pingali UR, Suresh Attili VS, Naidu Madireddy UR. Survival analysis in advanced non small cell lung cancer treated with platinum based chemotherapy in combination with paclitaxel, gemcitabine and etoposide. Asian Pac J Cancer Prev. 2013;14(8):4661-4666.

8. Ozdemir O, Ozdemir P, Veral A, et al. ERCC1 expression does not predict survival and treatment response in advanced stage non-small cell lung cancer cases treated with platinum based chemotherapy. Asian Pac J Cancer Prev. 2013;14(8):4679-4683.

9. Gurses N, Topcul M. The effect of abraxane on cell kinetic parameters of HeLa cells. Asian Pac J Cancer Prev. 2013;14(7):4229-4233.

10. Cui L, Liu XX, Jiang Y, et al. Phase II study on dose escalating schedule of paclitaxel concurrent with radiotherapy in treating patients with locally advanced non-small cell lung cancer. Asian Pac J Cancer Prev. 2014; 15(4):1699-1702.

11. Fang Y, Wang L, Xia GH, Shi MQ. Clinical investigation of efficacy of albumin bound paclitaxel plus platinum compounds as first-line chemotherapy for stage III/IV squamous non-small cell lung cancer. Asian Pac J Cancer Prev. 2014;15(17):7453-7457.

12. Aapro MS, Minckwitz GV. Molecular basis for the development of novel taxanes in the treatment of metastatic breast cancer. Eur J Cancer Suppl. 2008;6:3-11.

13. Desai N, Trieu V, Damascelli B, Soon-Shiong P. SPARC expression correlates with tumor response to albumin-bound paclitaxel in head and neck cancer patients. Transl Oncol. 2009;2(2):59-64.

14. Chen QQ, Ji XX, Zhou X, et al. Clinical observation of docetaxel or gemcitabine combined with cisplatin in the chemotherapy after surgery for stage II-III non-small cell lung cancer. Contemp Oncol. 2015; 19(4):323-326.

15. Schiller JH, Harrington D, Belani CP, et al. Comparison of four chemotherapy regimens for advanced non-small-cell lung cancer. $N$ Engl J Med. 2002;346(2):92-98.
OncoTargets and Therapy

\section{Publish your work in this journal}

OncoTargets and Therapy is an international, peer-reviewed, open access journal focusing on the pathological basis of all cancers, potential targets for therapy and treatment protocols employed to improve the management of cancer patients. The journal also focuses on the impact of management programs and new therapeutic agents and protocols on

\section{Dovepress}

patient perspectives such as quality of life, adherence and satisfaction. The manuscript management system is completely online and includes a very quick and fair peer-review system, which is all easy to use. Visit http://www.dovepress.com/testimonials.php to read real quotes from published authors. 